\title{
Problemas asociados al equilibrio en estructuras de membrana con bordes rígidos y cables
}

\author{
Equilibrium problems in membrane structures with rigid and \\ cable boundaries \\ G. Viglialoro $^{(*)}$, J. Murcia ${ }^{(* *)}$
}

RESUMEN

Este trabajo aborda el análisis del equilibrio de una membrana con bordes rígidos y cables de borde para la fase de pretensado. La idea de utilizar las membranas en aplicaciones como las pasarelas, una nueva tecnología que está siendo desarrollada en España, implica niveles más altos de responsabilidad y de esfuerzos, requiriendo así un análisis estructural ajustado. La membrana y sus bordes se identifican, respectivamente, a una superficie con curvatura de Gauss negativa y a curvas cuya curvatura depende de las características estructurales de los bordes. El equilibrio se expresa mediante problemas de contorno con ecuaciones en derivadas parciales, en términos de la forma de la membrana y de su tensor de esfuerzos, así como de la forma y las cargas de los bordes. En particular, el equilibrio asociado a un cable de borde lleva a una condición de frontera muy singular que dificulta un tanto el análisis. A partir de ello, caben dos enfoques complementarios, llamados problema directo y problema dual. Se definen y analizan ambos problemas de contorno, estudiando sus principales aspectos cualitativos. Asimismo, con el objetivo de obtener resultados prácticos, se propone un procedimiento general de resolución numérica para el problema directo.

445-24

Palabras clave: Membrana; Pasarela; Borde rígido; Cable de borde; Problema directo; Problema dual.
SUMMARY

This paper presents the equilibrium analysis of a membrane with rigid and cable boundaries for the so called prestressing phase. The idea of using membranes in Civil Engineering applications such as footbridges, a new technology being developed in Spain, implies higher structural responsibility and more accurate analysis procedure. The membrane and its boundary are respectively identified to a regular and negative gaussian curvature surface and a set of regular curves whose curvature depends on the structural elements, rigid or cable. Equilibrium is directly expressed by means of boundary differential problems, in terms of the membrane shape and its stress tensor. We must outline that membrane-cable interface equilibrium leads to take into account a singular condition that makes the problem more difficult. Therefore, starting from the equilibrium equations, two dual problems can be considered namely direct problem and dual problem. Both problems will be defined and analyzed, studying their principal qualitative aspects. Particularly, for the direct problem a numerical resolution procedure is proposed, in order to obtain practical results.

Key-words: Membrane; Footbridge; Rigid boundary; Boundary cable; Direct problem; Dual problem.

\footnotetext{
(*) Universidad de Cádiz (UCA), (España)

${ }^{(* *)}$ Instituto de Ciencias de la Construcción Eduardo Torroja (CSIC), España 
1. Esquema de pasarela de membrana con cables de borde.

\section{INTRODUCCIÓN}

Este trabajo estudia el equilibrio bidimensional y continuo (superficie) de una membrana, en la fase de pretensado (esto es, cuando la misma se encuentra preparada para la fase de servicio), con bordes rígidos y cables de borde. Así, se considera el equilibrio de la membrana directamente para la forma que resulta al ser pretensada.

La idea de utilizar membranas en nuevas aplicaciones de ingeniería civil como las pasarelas implica, con relación a otros usos, una gran responsabilidad estructural y mayores esfuerzos, debidos a las cargas de uso, a la forma muy rebajada de la membrana y al fuerte pretensado preciso para alcanzar la rigidez requerida. En consecuencia, hace falta un adecuado análisis bidimensional. En este contexto, se está desarrollando en España una nueva tecnología de pasarelas (1).

El problema del pretensado está relacionado con los bordes de la membrana, elementos unidimensionales definidos por líneas en el espacio. Los cables de borde son curvos, definidos por curvas que pertenecen a la superficie de la membrana y, para mantener todo a tracción, su curvatura ha de estar orientada hacia el exterior de la misma, tal como muestra la Figura 1. Los bordes rígidos, capaces de trabajar a flexión, admiten cualquier forma. Sin embargo, tal como se verá, el equilibrio en la interfaz cable-membrana implica que los cables de borde no puedan adoptar cualquier forma.

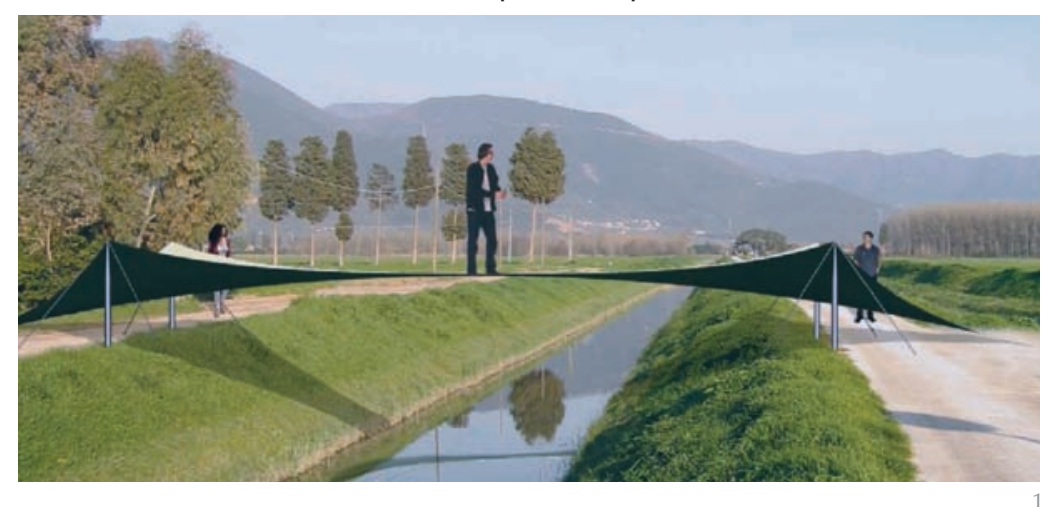

La consideración de bordes rígidos y cables de borde implica definir un problema más general que el analizado en el artículo (2). Como se verá, esto lleva a complicaciones importantes, tanto en términos de la formulación del problema como de su resolución.

Siguiendo la línea de dicho trabajo, los esfuerzos de la membrana se identifican con un tensor positivo de segundo orden y la forma con una superficie con curvatura de Gauss negativa. Así, es posible definir el equilibrio por medio de ecuaciones diferenciales en derivadas parciales, una de las cuales incluye los productos de variables asociadas a la forma de la membrana y al tensor de esfuerzos. Por tanto, si se fija el tensor de esfuerzos, puede considerarse el denominado problema directo $\mathrm{y}$, de modo complementario, el problema dual si lo que se fija es la forma de la membrana.

Con relación al equilibrio, si bien es cierto que las correspondientes ecuaciones en membranas son las mismas del análisis de los estados de membrana en láminas, ecuaciones bien conocidas (véanse, por ejemplo, la referencia (3)), también lo es que los problemas que se plantean son absolutamente diferentes, tal como se explica en el trabajo (2).

Como se ve en este trabajo, directamente del equilibrio por sí solo se pueden deducir bastantes cosas y, en particular, la forma de la membrana (cuando se fijan los esfuerzos), sin tener que considerar deformación alguna. Lo mismo sucede en el Método de la Densidad de Fuerzas, unidimensional (red superficial de cables en el espacio), basado exclusivamente en ecuaciones de equilibrio (4).

Una vez establecidas las ecuaciones de equilibrio interno para la membrana, es necesario definir las condiciones de frontera. Como se comentó antes, este trabajo es la continuación natural del artículo (2), porque generaliza el mismo problema considerando cables de borde además de bordes rígidos. Además, los cables de borde llevan a una formulación matemática de los problemas directo y dual que se complica sensiblemente con respecto al caso de bordes rígidos (véase, una vez más, la referencia (2)). Esto tiene mucho que ver con el hecho de que, precisamente, cable (1D) y membrana (2D) son estructuras singulares que no tienen rigidez propia (se la confiere la tracción).

Finalmente, una vez definidos rigurosamente los dos problemas, se estudian sus respectivos análisis cualitativos. Además, para el problema directo se presenta un procedimiento numérico capaz de proporcionar una buena estrategia de resolución. Este método se aplica a un caso real, la pasarela construida como prototipo (1), que se toma como referencia para evaluar los resultados. Por otro lado, se hacen algunas consideraciones sobre los aspectos matemáticos del problema dual.

\section{ECUACIONES DE EQUILIBRIO EN LA MEMBRANA}

Identifiquemos la membrana con una superficie $S$ con curvatura de Gauss negativa. En particular, $S$ es la gráfica de una función $z(x, y)$, definida en un dominio acotado $D$ del plano $x-y$, tal que $Z_{, x y} Z_{, y y}-Z^{2}{ }_{, x y}<0$ 
(la Figura 2 idealiza un elemento diferencial de membrana $d S$ y su correspondiente proyección $d D$ en el plano $x-y$, así como los relativos esfuerzos de membrana y proyectados, respectivamente $\tilde{N}_{\alpha \beta}$ y $N_{\alpha \beta}$, con $\left.\alpha, \beta=1,2\right)$

Como se sabe, el peso de la membrana es muy bajo y, en general, puede despreciarse para la fase de pretensado; más en particular para altos esfuerzos de pretensado como es el caso. Entonces, en esta fase no se considerará ninguna carga así que las ecuaciones de equilibrio serán homogéneas. Este aspecto no modifica la naturaleza del problema y es muy interesante desde el punto de vista del diseño (véase (2) para los detalles).

De esta forma, si $\sigma=N_{\alpha \beta}(\alpha, \beta=1,2)$ indica el tensor de esfuerzos proyectados, definido en $D$ (esto es, fuerza por unidad de longitud), las ecuaciones de equilibrio son [1]:

$N_{x x, x}+N_{x y, y}=0$ equilibrio en la dirección $x$, $\left\{N_{x y, x}+N_{y y, y}=0\right.$ equilibrio en la dirección $y$, $N_{x x} z_{x x}+2 N_{x y} z_{x y}+N_{y y} z_{y y}=0$ equilibrio en la dirección $z$,

Finalmente, la utilización de la función de Airy $H$, tal que [2]:

$H_{, x x}=N_{y y} H_{, x y}=-N_{x y} H_{, y y}=N_{x x^{\prime}}$

permite reducir el sistema [1] a la ecuación:

$H_{, x x} z_{, y y}-2 H_{, x y} z_{x y}+H_{, y y} z_{, x x}=0$

De esta forma, si se fija un tensor positivo $\sigma$ la función $z$ tiene que resolver una ecuación elíptica. Al contrario, una vez que se dé una superficie con curvatura de Gauss negativa, la función $H$ ha de resolver una ecuación hiperbólica (5).

Se pueden así definir dos problemas complementarios, el problema directo (se fijan los esfuerzos) y el problema dual (se fija la forma de la membrana).

\section{ECUACIONES DE EQUILIBRIO EN EL BORDE}

Con el objetivo de definir apropiadamente las condiciones de frontera para el problema directo y el problema dual, analicemos las correspondientes ecuaciones de equilibrio de borde.

Como ya se dijo, en este trabajo se analizarán ambos tipos de borde, rígido y cable; más en particular, en esta sección nos dedicaremos a estudiar las diferencias estructurales que los definen, concluyendo que la forma de un cable depende estrictamente de la forma de la membrana, mientras que el borde rígido puede asumir cualquier forma.

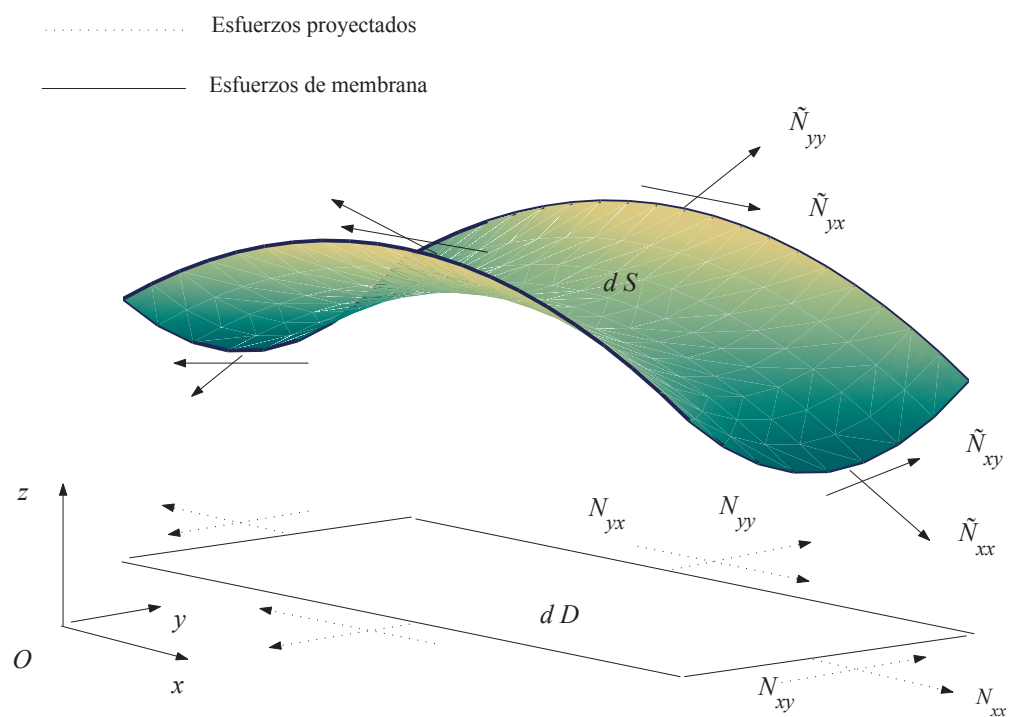

Identifiquemos el borde de la membrana con una curva espacial $C$; si $\tilde{N}_{\alpha \beta}(\alpha=1,2$ y $\beta=1,2)$ indica el tensor de esfuerzos sobre la membrana $S$ y $\tilde{f}=\left(\tilde{f}_{1}, \tilde{f}_{2}, \tilde{f}_{3}\right)$ la fuerza de borde repartida sobre $C$ (esto es, fuerza por unidad de longitud), resulta, al imponer el equilibrio, que $f$ es ortogonal al vector normal $N$ de la superficie $S$ a lo largo del contorno (para los detalles, véase la tesis (6)).

Además, siempre en (6), se comprueba que las ecuaciones de equilibrio para el borde tridimensional $C$ se reducen en las correspondientes para el borde plano $\Gamma=\partial D$, proyección horizontal de $C$. En este sentido, si $f=\left(f_{1}, f_{2}\right)$ indica la fuerza proyectada a lo largo de $\Gamma$ (fuerza por unidad de longitud), $\sigma=N_{\alpha \beta}$ el tensor de los esfuerzos proyectados y $n=\left(n_{1}, n_{2}\right)$ el vector normal (unitario) externo a $\Gamma$, el equilibrio de borde puede escribe como

$N_{\alpha \beta} n_{\beta}=f_{\alpha}(\operatorname{con} \alpha=1$ y 2$)$, sobre $\Gamma$

\subsection{Ecuaciones de equilibrio para el borde rígido}

En el caso en que se consideren sólo bordes rígidos, el equilibrio se reduce simplemente al sistema [4], tal y como se analiza en (2).

Por ejemplo, si la forma del borde rígido $\Gamma^{r}$ en el plano horizontal $x$ - $y$ está definido por rectas verticales, así como se hará más adelante para propósitos prácticos (véase la Figura 4), el sistema [4] se escribe como:

$\left\{\begin{array}{l}N_{x x}=f_{1} \text { sobre } \Gamma^{r} \\ N_{y y}=f_{2} \text { sobre } \Gamma^{r}\end{array}\right.$

De esta forma, el mismo sistema [4] permite obtener $f$ una vez que se fije $N_{\alpha \beta}$ y viceversa. 


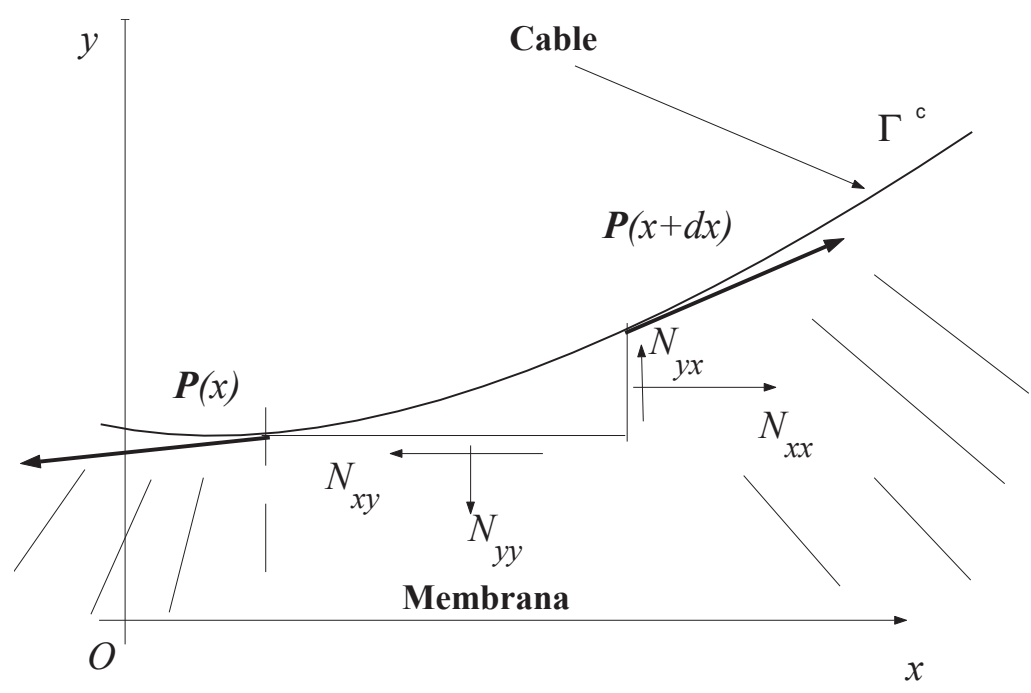

3. Interfaz cable-membrana ( $P$ representa la proyección de $\tilde{P}$ ).

\subsection{Ecuaciones de equilibrio para el cable de borde}

Si parte del contorno de la membrana está compuesto por un cable de borde, en el sistema [4] la fuerza correspondiente $f$ se expresa tanto en términos de la tensión como de la forma del mismo cable.

Identifiquemos el cable con una curva $C=(x, y(x), h(x))$ siendo $h(x)=z(x, y(x))$ la forma espacial del mismo cable,obtenida "levantando" el cable proyectado $y(x)$ por medio de la membrana $z(x, y)$. La tensión del cable es el vector tangente expresado por

$$
\tilde{P}=\frac{\tilde{P}}{\sqrt{1+y^{\prime}{ }^{2}+h^{\prime}}}\left(1, y^{\prime}, h^{\prime}\right),
$$

donde $\tilde{P}$ representa el valor de la tensión. De esta forma, la componente $x$ de $\tilde{P}$ es

$$
P_{x}=\frac{\tilde{P}}{\sqrt{1+y^{1^{2}+h^{\prime}}}},
$$

que utilizaremos a continuación. Con referencia a la Figura 3, es posible comprobar que el sistema [5]:

$\left\{P_{x}^{\prime}=N_{x y}-y^{\prime} N_{x x}\right.$,

$\left\{\begin{array}{l}x \\ \left(P_{x} y^{\prime}\right)^{\prime}=N_{y y}-y^{\prime} N_{x y},\end{array}\right.$

representa el equilibrio plano cable-membrana; este último sistema es un caso particular del sistema [4].

Por lo que se refiere al equilibrio en la dirección $z$, al tener en cuenta el sistema [5], se puede verificar que el equilibrio vertical cable-membrana se simplifica en la ecuación

$\left(P_{x} h^{\prime}\right)^{\prime}=-z_{, x} y^{\prime} N_{x x}+z_{, x} N_{x y}-y^{\prime} N_{x y} z_{, y}+z_{, y} N_{y y}$
Además, es posible comprobar que la última ecuación se expresa como:

$\left(P_{x} h^{\prime}\right)^{\prime}=P_{x}^{\prime} z_{, x}+\left(y^{\prime} P_{x}\right)^{\prime} z_{, y}$

Por otra parte, por medio de un simple cálculo (véase (6), para los detalles), se podría argumentar que la ecuación [6], considerando una vez más también el sistema [5], es equivalente a

$z_{, x x}+2 y^{\prime} z_{, x y}+y^{\prime 2} z_{, y y}=0$

Esta última relación, que representa la ecuación de compatibilidad entre el cable y la membrana, implica que la fuerza repartida $\tilde{f}$ no sólo es ortogonal a la normal $\mathrm{N}$ a la superficie $S$ sino que pertenece al plano osculador de la curva $C$.

Para comprobarlo, si $N$ es el vector unitario normal a $S$, y $t$ y $n$ aquellos tangente y normal al cable $C$, es suficiente imponer que los vectores $t$ y $n$ son ortogonales a $N$. Siendo $t$ proporcional a $\left(1, y^{\prime}, z_{, x}+z_{, y} y^{\prime}\right), n$ a $\left(0, y^{\prime \prime}, z_{, x x}+\right.$ $\left.2 y^{\prime} z_{, x y}+y^{\prime} z_{, y y}+y^{\prime \prime} z_{, y}\right)$ y $N$ a $\left(z_{, x} z_{, y},-1\right)$, se debe de cumplir $z_{, x x}+2 y^{\prime} z_{, x y}+y^{\prime} z_{, y y}=0$

En este trabajo, al tenerse en cuenta los cables de borde, se utilizará el siguiente sistema para expresar el equilibrio cable-membrana [8]:

$\left\{\begin{array}{l}\left.P_{x}^{\prime}=N_{x y}-y^{\prime} N_{x x} \text { (equilibrio en la dirección } x\right) \\ \left.\left(P_{x} y^{\prime}\right)=N_{y y}-y^{\prime} N_{x y} \text { (equilibrio en la dirección } y\right) \\ \left.\left(P_{x} h^{\prime}\right)=P^{\prime} z_{x}+\left(y^{\prime} P_{x}\right)^{\prime} z_{y} \text { (equilibrio en la dirección } z\right)\end{array}\right.$

Al revés, la tesis referenciada en [8] analiza el mismo problema partiendo del sistema anterior en el que se sustituye su última ecuación por la expresión [7].

\section{EL PROBLEMA DIRECTO: DEFINICIÓN Y PROPIEDADES}

En esta sección definiremos y analizaremos el problema directo, proponiendo, además, un procedimiento numérico para el cálculo aproximado de su solución.

\section{Problema directo}

Con referencia al equilibrio expresado por el sistema [1], sea $N_{\alpha \beta}$ un tensor positivo $y$ simétrico del segundo orden tal que

$\left\{\begin{array}{l}N_{x x, x}+N_{x y, y}=0 \\ N_{x y, x}+N_{y y, y}=0\end{array} \leftrightarrow \sum_{\beta=1}^{2} N_{\alpha \beta, \beta}=0\right.$

$\alpha=1,2$ en un domino acotado $D$ del plano $x-y$.

Hallar la superficie $z$, definida en $D$, de manera que 
$\left\{\begin{array}{l}z_{x x} N_{x x}+2 z_{x y} N_{x y}+z_{, y y} N_{y y}=0 \text { en } D, \\ z=g \text { sobre } \Gamma^{r} \\ \left(P_{x} h^{\prime}\right)^{\prime}=P_{x}^{\prime} z_{, x}+\left(y^{\prime} P_{x}\right)^{\prime} z_{, y} \text { sobre } \Gamma^{c}\end{array}\right.$

siendo $h(x)=z(x, y(x))$ la forma espacial del cable, $\Gamma^{r}$ la parte del contorno de $D$ compuesto por bordes rígidos, $\Gamma^{c}$ la correspondiente a los cables de borde, y $g(y)$ el valor de $z$ a lo largo del mismo borde rígido $\Gamma^{r}$.

Debido a la segunda condición de frontera, el anterior no es un típico problema elíptico con condición usual de Dirichlet o DirichletNeumann (véanse $(7,8)$ ). En la tesis $(6)$, se comprueba por medio de rigurosos resultados matemáticos la unicidad de la solución. En el siguiente apartado, se propondrá un método numérico útil para el cálculo aproximado de la solución; no obstante, hay que matizar que argumentar la existencia del mismo sistema [9] no parece ser del todo inmediato.

Por supuesto, si el borde de la membrana es totalmente rígido (esto es $\Gamma^{c}=\varphi$ ), el sistema [9] se reduce a un problema de Dirichlet, ya profundizado en el trabajo (2).

Antes de desarrollar el método de resolución del problema directo, es oportuno matizar algunos aspectos relativos al sistema [8]. En particular, tal y como manifiestan las ecuaciones de equilibrio que lo definen, es posible calcular, a partir de los esfuerzos $N_{\alpha \beta}$ la forma del cable de borde proyectado $y(x)$ que, juntamente con el borde rígido $\Gamma^{r}$ permite fijar el dominio $D$ (véase Figura 4). De la misma forma, es posible calcular $P_{x^{\prime}}$ componente de la tensión del cable en la dirección $x$, a partir de un cierto valor $P_{x}^{0}$ conocido. No obstante, la forma espacial del cable (expresada por la función $h(x)$ es una incógnita del problema, que se obtiene al conocer la forma de la membrana $z(x, y)$.

\subsection{Método de resolución para el problema directo}

Las condiciones $\sum_{\beta=1}^{2} N_{\alpha \beta, \beta}=0,(\alpha=1,2)$ permiten escribir el sistema [9] en la siguiente forma divergencial [10]:

$$
\left\{\begin{array}{l}
\operatorname{div}(\sigma \cdot \nabla z)=0 \text { en } D \\
z=g \text { sobre } \Gamma^{r} \\
\left(P_{x} h^{\prime}\right)^{\prime}=P_{x}^{\prime} z_{, x}+\left(y^{\prime} P_{x}\right)^{\prime} z_{, y} \text { sobre } \Gamma^{c}
\end{array}\right.
$$

Este último sistema será el punto de partida para el análisis del problema directo.

A continuación, utilizando el Método de los Elementos Finitos (véanse, por ejemplo, $(9,10)$, se propondrá una técnica capaz de proporcionar la solución aproximada del sistema [10].

Reescribamos el problema [10], considerando por un lado el sistema reducido (con condición de frontera clásica, de tipo Dirichlet) [11]:

$\left\{\begin{array}{l}\operatorname{div}(\sigma \cdot \nabla z)=O \text { en } D \\ z=g \text { sobre } \Gamma^{r} \\ z=h \text { sobre } \Gamma^{c}\end{array}\right.$

y, por otro, la ecuación [12]:

$\left(P_{x} h^{\prime}\right)^{\prime}=P_{x}^{\prime} z_{x}+\left(y^{\prime} P_{x}\right)^{\prime} z_{, y}$ sobre $\Gamma^{c}$

Una vez fijada una malla para $D$, indiquemos con $n_{t}$ el número total de nodos en $D, n_{r}$ el número total de nodos sobre $\Gamma^{r}$ y $n_{c}$ el número total de nodos sobre $\Gamma^{c}$. Si con $N_{j}$ nos referimos a las funciones de forma lineales, reemplazando las correspondientes aproximaciones $z \cong \sum_{j=1}^{n_{t}} z_{j} N_{j}$ y $h^{\sim} \sum_{j=1}^{n_{c}} h_{j} N_{j}$ en el sistema [11], se obtiene el siguiente sistema lineal[13].

$$
\left(\begin{array}{ccc}
K & A_{r}^{T} & A_{c}^{T} \\
A_{r}^{T} & 0 & 0 \\
A_{c}^{T} & 0 & 0
\end{array}\right)\left(\begin{array}{l}
z \\
\lambda_{r} \\
\lambda_{c}
\end{array}\right)=\left(\begin{array}{l}
0 \\
g \\
h
\end{array}\right)
$$

En la última ecuación matricial, $z=\left(z_{1}, \ldots, z_{t}\right)$ $\boldsymbol{g}=\left(g_{1}, \ldots, g_{r}\right)$ y $\boldsymbol{h}=\left(h_{1}, \ldots, h_{c}\right)$ indican el vector nodal de $z$ en $D$, de $g$ sobre $\Gamma^{c}$ de $h$ sobre $\Gamma^{c}$, $k$ la matriz de rigidez, $A_{\gamma}$ y $A_{C}$ las matrices de los Multiplicadores de Lagrange, y $\lambda_{\gamma}$ y $\lambda_{c}$ los vectores de los Multiplicadores de Lagrange.

Sea, ahora, z la solución del sistema [13]: puede escribirse

$z=\mathcal{H} h+\mathcal{G g}$

siendo

$\mathcal{H} \in M_{\mathrm{n}_{v} \mathrm{n}_{\mathrm{c}}}(\mathcal{R})$ y $\mathcal{G} \in M_{\mathrm{n}_{v} \mathrm{n}_{c}}(\mathcal{R})$

Además, teniendo en cuenta la ecuación [12], puede considerarse la ecuación $n_{c}$ - dimensional, definida por

$$
\begin{array}{r}
\int_{\Gamma^{c}}\left[P^{\prime}{ }_{x} z_{, x}+\left(y^{\prime} P_{x}\right)^{\prime} z_{, y}-\left(P_{x} h^{\prime}\right)^{\prime}\right] N_{i} d \Gamma^{c}=0 \quad[15] \\
i=1,2,3 \ldots, n_{c}
\end{array}
$$

Reemplazando, igual que antes, las aproximaciones $z \cong \sum_{j=1}^{n_{i}} z_{j} N_{j}$ y $h \cong \sum_{j=1}^{n_{i}} h_{j} N_{j}$ e integrando por partes, se obtiene la expresión:

$$
\begin{aligned}
& \sum_{j=1}^{n_{t}} z_{j} \int_{\Gamma^{c}}\left[N_{j, x} P^{\prime}{ }_{x}+N_{j, y}\left(y^{\prime} P_{x}\right)^{\prime}\right] N_{i} d \Gamma^{c}+\sum_{j=1}^{n_{c}} h_{j} \int_{\Gamma^{c}} P_{x} N^{\prime}{ }_{j} N^{\prime}{ }_{i} d \Gamma^{c}=0, \\
& \text { Finalmente, definiendo, } \\
& i=1,2,3 \ldots, n_{c}
\end{aligned}
$$

$$
\begin{aligned}
& M_{i j}=\int_{\Gamma^{c}}\left[N_{j, x} P^{\prime}{ }_{x}+N_{j, y}\left(y^{\prime} P_{x}\right)^{\prime}\right] N_{i} d \Gamma^{c} \\
& \text { y } W_{i j}=\int_{\Gamma^{c}} P_{x} N^{\prime}{ }_{j} N^{\prime}{ }_{i} d \Gamma^{\prime}
\end{aligned}
$$


se conviene al siguiente sistema

$\mathcal{M} z+\mathcal{W h}=0$

en el que

$\mathcal{M} \in M_{\mathrm{n}_{\sigma} \mathrm{n}_{\mathrm{t}}}(\mathbb{R})$ y $\mathcal{W} \in M_{\mathrm{n}_{c} \mathrm{n}_{c}}(\mathbb{R})$

Comparando los sistemas [14] y [16], se obtiene la siguiente expresión para la solución:

$h=(\mathcal{M H}+\mathcal{W})^{-1} \mathcal{M G g}$

siendo $h$ el vector nodal de $z$ a lo largo de $\Gamma^{\mathrm{c}}$, que nos proporciona una aproximación tridimensional de la forma del cable.

De la misma manera, es posible obtener la solución $z$,

$z=\mathcal{H} h+\mathcal{G g}$

siendo, ahora, $h$ el vector nodal de $z$ en $D$, que nos proporciona una aproximación tridimensional de la forma de la membrana.

\subsection{Un ejemplo concreto de problema directo}

Apliquemos el procedimiento numérico previamente analizado para resolver el caso de la pasarela peatonal construida en España. Es importante comentar que el caso presentado en el ejemplo que sigue es casi igual, pero no exactamente, al llevado a cabo en la realidad. Ello es debido a que, recordando que se trata de encontrar la forma de la membrana (y de los cables de borde), el método de cálculo para ello en el caso real fue aproximado. Así, las formas por una y otra vía no pueden ser idénticas y, en consecuencia, ambos casos tampoco pueden serlo, solamente bastante aproximados.

En efecto, en el caso real, se realizó una aproximación analítica, ajustando expresiones para los esfuerzos y las formas de membrana (superficie) y cables de borde a todas las ecuaciones de equilibrio ya vistas; cuya solución, aproximada pero bastante precisa, implicaba valores de los esfuerzos de membrana en las direcciones de los ejes casi constantes pero no exactamente véase (1).
Por su parte, aquí se aplica el citado procedimiento y, como de todos modos no puede obtenerse un caso idéntico, se simplifica fijando esfuerzos de membrana constantes, muy aproximados a los anteriores (valores diferentes en las direcciones de los ejes).

\section{Datos para el ejemplo}

Todas las longitudes ( $x, y, z$, así como las longitudes en las figuras) se expresan en metros.

El dominio $D$ tiene la forma mostrada en la Figura 4:

$D:=\left\{(x, y) \in \mathbb{R}^{2}\right.$ tal que $\left.-a \leq x \leq a y-y(x) \leq y \leq y(x)\right\}$ siendo $a=5 \mathrm{~m}$ e $y=x$ obtenida resolviendo el sistema [5].

Las condiciones sobre el borde rígido y el tensor de esfuerzos son:

$\left\{\begin{array}{l}g(y)=\frac{17}{800} y^{2}\left(11-\frac{1}{6} y^{2}\right) \text { forma de } z \text { a lo largo de } \Gamma^{\prime} \\ N_{x x}=10 \mathrm{kN} / \mathrm{m}, N_{x y}=0 \mathrm{kN} / \mathrm{m}, N_{y y}=4 \mathrm{kN} / \mathrm{m} .\end{array}\right.$

De esta forma, el sistema [5] se resume en $\left\{\begin{array}{l}P_{x}^{\prime}=-10 y^{\prime} \\ \left(P_{x} y^{\prime}\right)^{\prime}=4\end{array}\right.$

$\varepsilon_{+}: \frac{8}{605} x^{2}+\frac{4}{121}\left(y-\frac{13}{2}\right)^{2}=1$ y $\varepsilon_{-}: \frac{8}{605} x^{2}+\frac{4}{121}\left(y+\frac{13}{2}\right)^{2}=1$.

Integrando, usando la simetría e imponiendo $y( \pm 5)=2$ y $y(0)=1$ y $y( \pm 5)=-2$ y $y(0)=1$, se obtienen las dos elipses, $\varepsilon_{+}$y $\varepsilon_{\text {. }}$

Escogiendo las ramas adecuadas de $\varepsilon_{+}$y $\varepsilon_{\text {. }}$ (gráfica (a) de la Figura 5), es natural fijar e

$D:=\left\{(x, y) \in \mathbb{R}^{2}\right.$ tal que $\left.-a \leq x \leq a y-y(x) \leq y \leq y(x)\right\}$

$y(x)=\frac{13}{2}-\sqrt{\frac{121}{4}-\frac{2}{5} x^{2}}$

(la gráfica (b) de la Figura 5 representa una malla compuesta por 477 nodos).

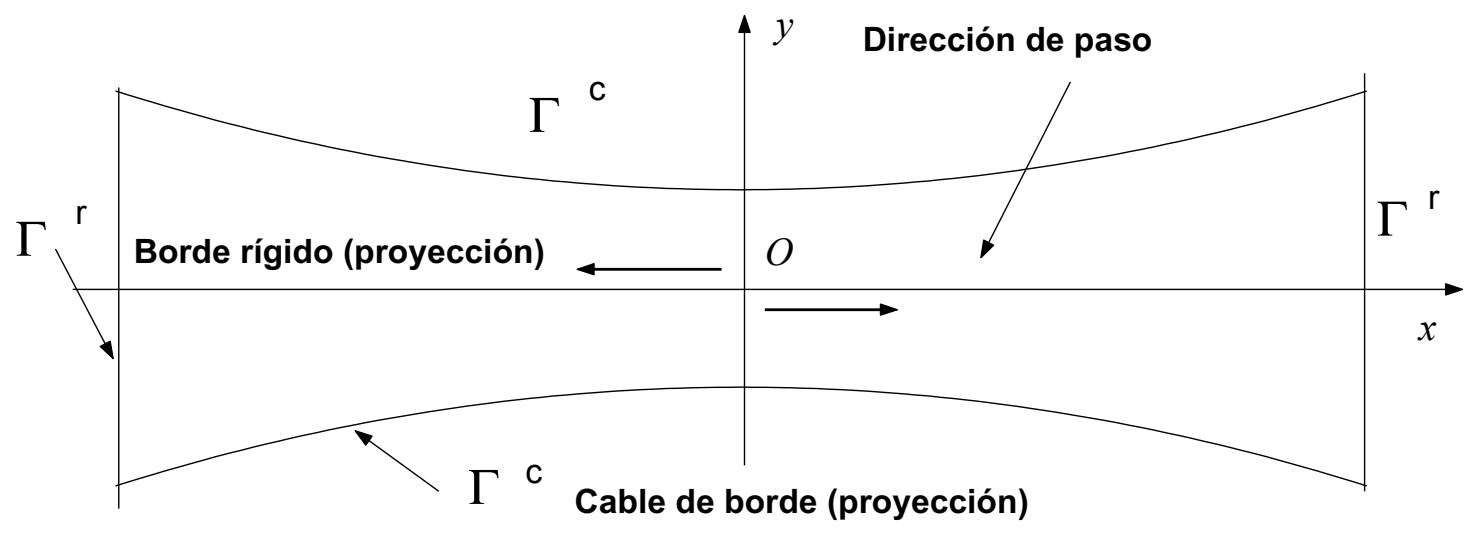


En estas condiciones, hay que resolver el sistema

$$
\begin{aligned}
& 10 z_{, x x}+4 z_{, y y}=0 \text { en } D, \\
& z=\frac{17}{800} y^{2}\left(11-\frac{1}{6} y^{2}\right) \text { sobre } x=\mp 5\left(\text { i.e } \Gamma^{\mathrm{c}}\right), \\
& z=h \text { sobre } y(x)=\mp\left(\frac{13}{2}-\sqrt{\frac{121}{4}-\frac{2}{5} x^{2}}\right)\left(\text { i.e } . \Gamma^{\mathrm{c}}\right), \\
& \left(P_{x} h^{\prime}\right)^{\prime}=P^{\prime}{ }_{x} z_{, x}+\left(y^{\prime} P_{x}\right)^{\prime} z_{, y} \quad \text { sobre } y(x)=\mp\left(\frac{13}{2}-\sqrt{\left.\frac{121}{4}-\frac{2}{5} x^{2}\right)} \quad \text { (i.e. } \Gamma^{c}\right)
\end{aligned}
$$

Método Iterativo del Punto Fijo $(7,8)$, ajustando "paso a paso" una cierta forma inicial $h_{0}$, de manera que en el límite se satisfagan todas las ecuaciones del mismo sistema [9].
5. Dominio D escogido para el ejemplo del problema directo.

Sub-figura (a): Cables de borde (ramas de elipse).

Sub-figura (b): Malla de 477 nodos para el dominio $\mathrm{D}$.
Las gráficas (a) de las Figuras 6 y 7 representan los resultados numéricos obtenidos, tomando como punto más bajo de la membrana (esto es, el extremo $(-5,0)$ el origen de las medidas. Estos resultados han sido calculados ejecutando un programa en MATLAB, basado en la utilización de elementos cuadriláteros lineales (es posible encontrar los detalles del método en la tesis (6).

Como ya se ha comentado anteriormente, el caso ahora analizado no se corresponde a aquél real estudiado en el trabajo (1). A pesar de ello, comparando sendas soluciones obtenidas, es posible confirmar que las mismas son del todo coherentes. De hecho, por ejemplo, la flecha del cable calculada en el caso real es de 0,31 $\mathrm{m}$, contra el valor de $0,30 \mathrm{~m}$ obtenido por medio del método numérico aquí propuesto.

Aparte ello, también es posible analizar la componente de la tensión del cable $P_{x}$.

En fecto, debido a la relación

$P_{x}^{\prime}=-10 y^{\prime}$

imponiendo por ejemplo

$P_{x}^{0}=P_{x}(0)=55 k N$, se obtiene la expresión

$P_{x}=10 \sqrt{\frac{121}{4}-\frac{2}{5} x^{2}}$

Finalmente, cabe subrayar que el procedimiento utilizado presenta una cierta inestabilidad numérica. De hecho, la gráfica (a) de la Figura 6 muestra que la solución numérica para el cable $h=h(x)$ varía en términos del número de nodos utilizados; en particular, no es posible observar un comportamiento estrictamente monótono para la solución. Esto parece estar relacionado con la formulación del problema y no tanto con la aproximación de elementos finitos empleados. En efecto, analizando en detalle las ecuaciones de equilibrio, podemos notar que en el sistema [9] se fuerza a la incógnita $z$ a que verifique en el borde una ecuación del mismo orden que la que ha de cumplirse en el interior del dominio, siendo lo usual que en la frontera la incógnita cumpla condiciones de órdenes inferiores.

Una posible alternativa para la resolución, sobre la que los autores están trabajando, se basa en buscar la forma final del cable $h$ mediante el

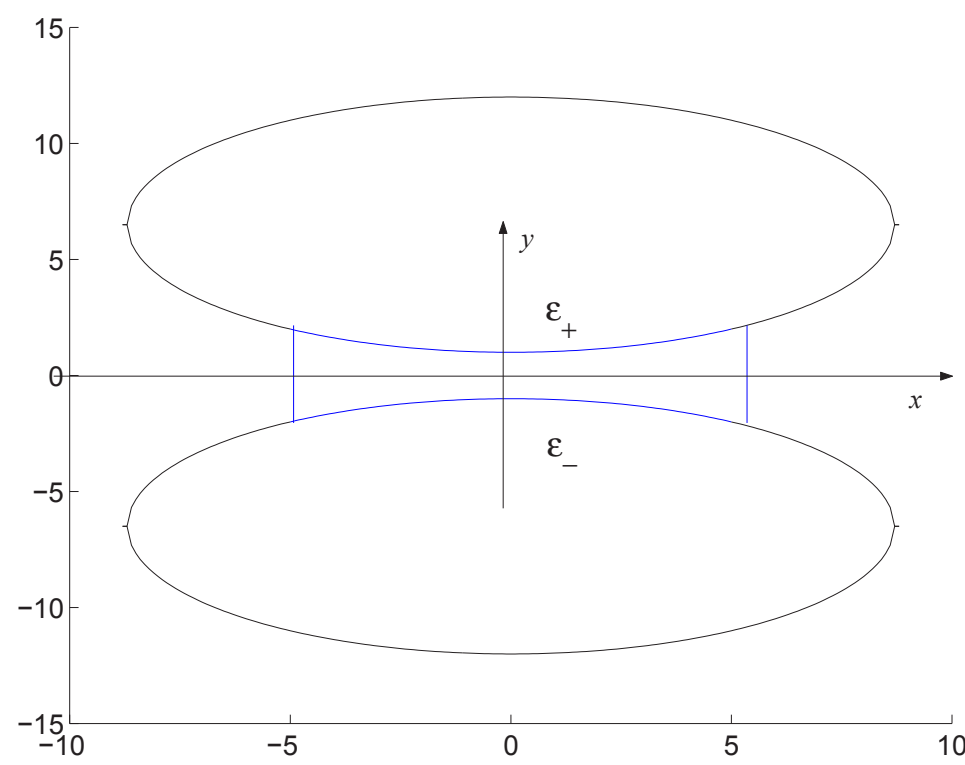

$5 a$

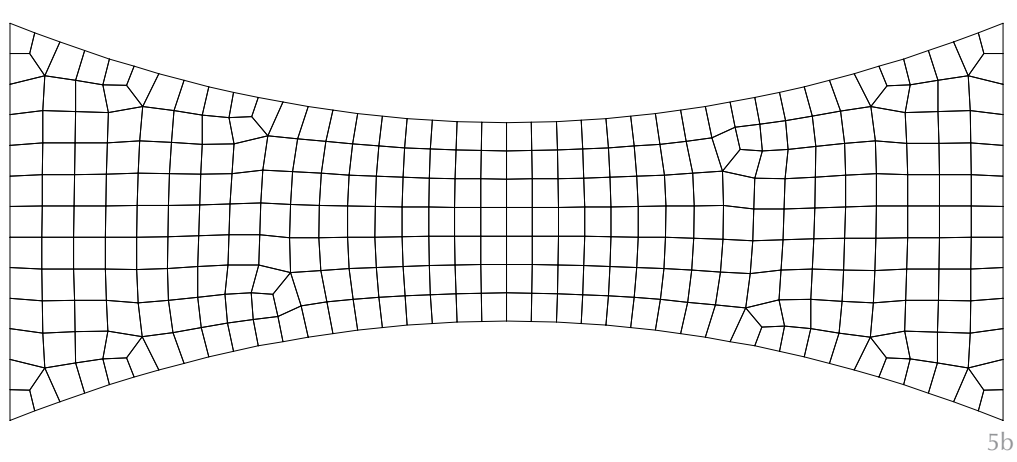

\section{PROBLEMA DUAL: DEFINICIÓN Y PROPIEDADES}

En este apartado se definirá y analizará el problema dual.

\section{Problema dual}

Con referencia al equilibrio expresado por el sistema [1], sea $S=(x, y, z(x, y))$ una superficie con curvatura de Gauss negativa. Al resolver la ecuación [7] se obtiene una función $y(x)$ que define el cable de borde proyectado, indicado con $\Gamma^{c}$.

Si con $f=f(y)$ se denota la fuerza sobre el borde rígido $\Gamma^{r}$, hallar el tensor de esfuerzos $\sigma=N_{\alpha \beta}$ en el dominio $D$, tal que 

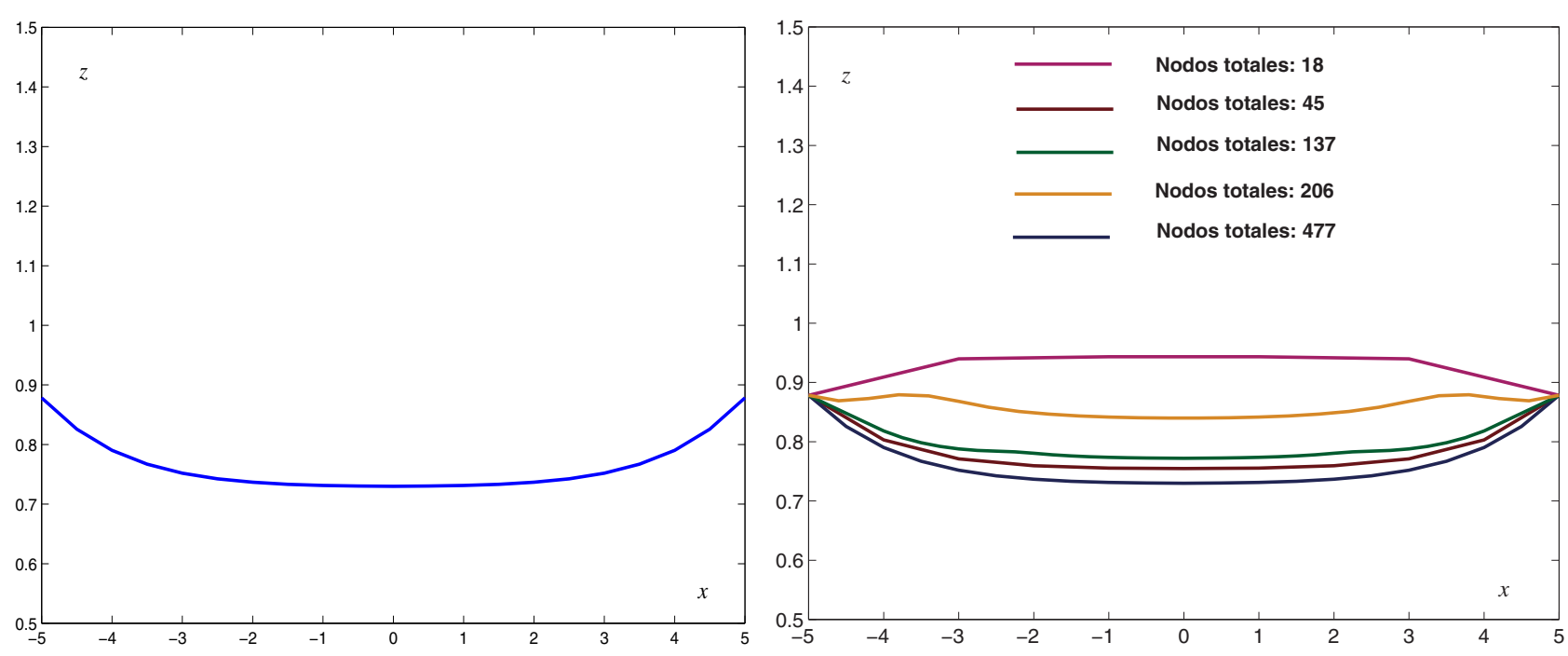

$6 a$

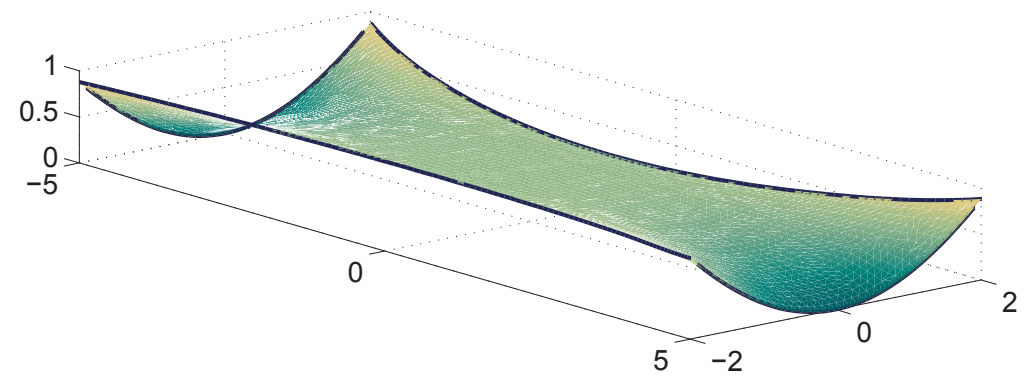

$\mathrm{m}$

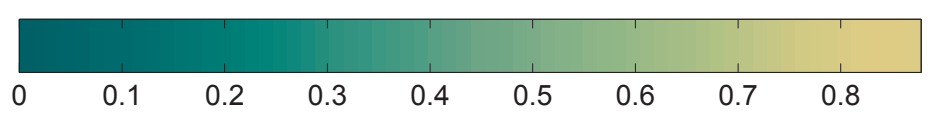

6a. Solución numérica calculada usando 137 nodos; proyección vertical del cable.

6b. Solución numérica de $h=h(x)$; proyección vertical del cable en términos del número de nodos.

7. Pasarela real y gráfica de su forma calculada numéricamente

Sub-figura (a): Solución numérica $z=z(x, y)$.

$$
y^{\prime}(x)=\frac{-z_{, x y} \pm \sqrt{z_{, x y}^{2}-z_{, x x} z_{, y y}}}{z_{, y y}},
$$

que se reduce a

$z_{, x x}+2 y^{\prime} z_{, x y}+y^{\prime 2} z_{, y y}=0$

Como ya se comentó, esta relación, junta con las del sistema [5], es equivalente a

$\left(P_{x} h^{\prime}\right)^{\prime}=P_{x}^{\prime} z_{x}+\left(y^{\prime} P_{x}\right)^{\prime} z_{, y}$

Todo ello implica que en el problema dual las curvas que representan los cables de borde coinciden con las curvas características de la ecuación. En este sentido, deberíamos considerar un problema hiperbólico con condiciones de frontera definidas a lo largo de sus curvas características. Tal y como se detalla en (5), no es siempre posible garantizar la existencia, unicidad y estabilidad de la solución para este tipo de problemas.

Por tanto, recordando también los casos de bordes rígidos (2), en el problema dual las ecuaciones de equilibrio pueden no ser suficientes para calcular la distribución de esfuerzos que equilibra la forma previamente fijada; al revés de lo que ocurre en el problema directo. En otras palabras, el problema dual no es siempre isostático. Ahora bien, desde el punto de vista estructural, está claro que si se consideraran también las ecuaciones constitutivas y de compatibilidad no nos enfrentaríamos a este tipo de singularidad, obteniendo un problema hiperbólico coherente y bien planteado.

Además, al analizar las curvas características de la ecuación de equilibrio de membrana

$H_{, x x} Z_{, y y}-2 H_{, x y} z_{, x y}+H_{, y y} Z_{, x x}=0$

se obtiene la ecuación diferencial (ordinaria)
En fin, con relación al problema dual, conviene indicar que en (6) se ofrece una casuística de ejemplos analíticamente resolubles para borde rígido, a partir de algunos resultados propios de la ecuación de ondas. 


\section{RESUMEN Y CONCLUSIONES}

En este trabajo se ha analizado el equilibrio de una membrana, para la fase de pretensado, en el caso general en que sus bordes están compuestos por elementos rígidos y cables. Se han formulado las ecuaciones de equilibrio sobre la superficie y los bordes de la membrana mediante variables asociadas a la forma y a los esfuerzos de todos ellos. Así, según el tipo de variables fijadas, se definen dos problemas: el problema directo y el problema dual.

Este tipo de análisis riguroso, en el continuo bidimensional, está asociado a los fuertes requisitos estructurales de una nueva tecnología de pasarelas con estructura de membrana que está siendo desarrollada en España.

Un aspecto relevante del trabajo es el análisis bidimensional en los bordes, particularmente en el caso de cables, cuya forma, al contrario que los bordes rígidos, no puede fijarse arbitrariamente a priori.

Hay que destacar, en particular, que el equilibrio en la interfaz cable-membrana conduce a una ecuación singular, que implica considerar un del todo inusual y complejo problema diferencial de contorno. Esto se relaciona con el hecho de que ahí interaccionan precisamente las dos estructuras singulares sin rigidez propia, cable (1D) y membrana (2D), que no pueden adoptar cualquier forma para estar en equilibrio.
Más precisamente, si por un lado es cierto que el problema directo se basa en una ecuación diferencial elíptica, por otro la condición de contorno asociada no es ni de tipo Dirichlet ni de tipo Dirichlet-Neumann. En este sentido, aunque es posible argumentar de forma rigurosa la unicidad de la solución, no es inmediato demostrar su existencia. Sin embargo, desde un punto de vista práctico, puede proponerse un procedimiento numérico, como aquí se ha hecho empleando el Método de los Elementos Finitos, para la construcción de la solución.

Además, se ha resuelto un caso particular de problema directo mediante el método numérico y se han comparado sus resultados con un caso real de referencia. La comparación muestra valores similares y coherentes. Por otro lado, en el método aquí propuesto es posible observar una cierta inestabilidad, debida a la formulación intrínseca del problema.

Finalmente, el problema dual es de tipo hiperbólico, para el cual no siempre es posible proporcionar una solución, única y estable; esto es, en el problema dual no siempre existe isostatismo. Pero, además, cuando existen cables de borde, la condición de equilibrio entre cable y membrana implica que los cables de borde coinciden con las curvas características de la ecuación diferencial. Por tanto, el problema dual con condiciones de frontera definidas a lo largo de dichos cables no es en general matemáticamente admisible.

\section{BIBLIOGRAFÍA}

(1) Murcia J.: "Tecnología de pasarelas con estructura de membrana". Informes de la Construcción; vol. 59, n. ${ }^{\circ}$ 507, 21-31, 2007.

(2) Viglialoro G.; Murcia J.; Martínez F.: "Problemas asociados al equilibrio en estructuras de membrana con bordes rígidos". Informes de la Construcción; vol. 61, n. ${ }^{\circ}$ 516, 57-66, 2009.

(3) Timoshenko S.; Woinowsky-Krieger S.: Theory of Plates and Shells. McGraw-Hill, Inc., New York, 1959.

(4) Linkwitz K.: "About formfinding of double-curved structures". Engineering Structures; vol. 21, pp. 709-718, 1999.

(5) Hörmander L.: Linear Partial Differential Operators. Springer, Berlín, 1964.

(6) Viglialoro G.: Análisis matemático del equilibrio en estructuras de membrana con bordes rígidos y cables. Pasarelas: forma y pretensado. Tesis doctoral, Universidad Politécnica de Cataluña. ISBN: 978-84-690-6482-5, 2006. Depósito legal: B.33064-2007. Enlace: http://www.tesisenxarxa.net/TDX-0515107-100745/.

(7) Brezis H.: Análisis funcional: teoría y aplicaciones. Madrid, Alianza Editorial, 1983.

(8) Gilbarg L.; Trudinger N. S.: Elliptic Partial Diferential Equations of Second Order. Springer, 1998.

(9) Hughes T. J. R.: The Finite Element Method. Linear Static and Dynamic Finite Element Analysis. Mineola, New York Dover Publications, 1987.

(10) Zienkiewicz O. C.; Taylor R. L.: The Finite Element Method. Butterworth Heinemann, 2000. 